\title{
Front Matter: Volume 7073
}

, "Front Matter: Volume 7073," Proc. SPIE 7073, Applications of Digital Image Processing XXXI, 707301 (3 October 2008); doi: 10.1117/12.815022

SPIE Event: Optical Engineering + Applications, 2008, San Diego, California, United SPIE. States 


\title{
PROCEEDINGS OF SPIE
}

\section{Applications of Digital Image Processing XXXI}

\author{
Andrew G. Tescher \\ Editor \\ 11-14 August 2008 \\ San Diego, California, USA \\ Sponsored and Published by \\ SPIE
}

Volume 7073 
The papers included in this volume were part of the technical conference cited on the cover and title page. Papers were selected and subject to review by the editors and conference program committee. Some conference presentations may not be available for publication. The papers published in these proceedings reflect the work and thoughts of the authors and are published herein as submitted. The publisher is not responsible for the validity of the information or for any outcomes resulting from reliance thereon.

Please use the following format to cite material from this book:

Author(s), "Title of Paper," in Applications of Digital Image Processing XXXI, edited by

Andrew G. Tescher, Proceedings of SPIE Vol. 7073 (SPIE, Bellingham, WA, 2008) Article CID Number.

ISSN 0277-786X

ISBN 9780819472939

Published by

SPIE

P.O. Box 10, Bellingham, Washington 98227-0010 USA

Telephone +1 3606763290 (Pacific Time) · Fax +1 3606471445

SPIE.org

Copyright (C 2008, Society of Photo-Optical Instrumentation Engineers

Copying of material in this book for internal or personal use, or for the internal or personal use of specific clients, beyond the fair use provisions granted by the U.S. Copyright Law is authorized by SPIE subject to payment of copying fees. The Transactional Reporting Service base fee for this volume is $\$ 18.00$ per article (or portion thereof), which should be paid directly to the Copyright Clearance Center (CCC), 222 Rosewood Drive, Danvers, MA 01923. Payment may also be made electronically through CCC Online at copyright.com. Other copying for republication, resale, advertising or promotion, or any form of systematic or multiple reproduction of any material in this book is prohibited except with permission in writing from the publisher. The CCC fee code is $0277-786 \mathrm{X} / 08 / \$ 18.00$.

Printed in the United States of America.

Publication of record for individual papers is online in the SPIE Digital Library.

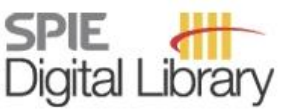

SPIEDigitallibrary.org

Paper Numbering: Proceedings of SPIE follow an e-First publication model, with papers published first online and then in print and on CD-ROM. Papers are published as they are submitted and meet publication criteria. A unique, consistent, permanent citation identifier (CID) number is assigned to each article at the time of the first publication. Utilization of CIDs allows articles to be fully citable as soon they are published online, and connects the same identifier to all online, print, and electronic versions of the publication. SPIE uses a six-digit CID article numbering system in which:

- The first four digits correspond to the SPIE volume number.

- The last two digits indicate publication order within the volume using a Base 36 numbering system employing both numerals and letters. These two-number sets start with 00, 01, 02, 03, 04, 05, $06,07,08,09,0 A, 0 B \ldots 0 Z$, followed by $10-12,20-2 Z$, etc.

The CID number appears on each page of the manuscript. The complete citation is used on the first page, and an abbreviated version on subsequent pages. Numbers in the index correspond to the last two digits of the six-digit CID number. 


\section{Contents}

xi Conference Committee

\section{SESSION 1 IMPLEMENTATION AND PROCESSING SCENARIOS I}

707302 An improved image scene registration with wavelet preprocessing [7073-01]

E. P. Lam, Thales Raytheon Systems (United States)

707303 Pattern recognition of an implicitly given target [7073-02]

P. M. Aguilar-González, V. Kober, Ctr. de Investigación Científica y de Educación Superior de Ensenada (Mexico)

707304 Wavelet domain denoising by using the universal hidden Markov tree model [7073-03] F. Li, D. Fraser, X. Jia, A. Lambert, Australian Defence Force Academy, Univ. of New South Wales (Australia)

\section{SESSION 2 DESIGN AND IMPLEMENTATION OF COLOR TRANSFORMATIONS}

707306 CIELAB to CMYK color conversion in the transform domain for JPEG compressed images [7073-06]

Y. Qiao, J. L. Mitchell, N. Rijavec, A. T. Hinds, InfoPrint Solutions Co. (United States)

707307 Lifting-based reversible color transformations for image compression [7073-07]

H. S. Malvar, G. J. Sullivan, S. Srinivasan, Microsoft Corp. (United States)

707308 Faster color conversion via lookup table mesh dimension management [7073-08] N. Rijavec, InfoPrint Solutions Co. (United States)

707309 Continued fractions, diophantine approximations, and design of color transforms [7073-09] Y. A. Reznik, Qualcomm Inc. (United States)

7073 OA Complex color management using optimized nonlinear three-dimensional look-up tables [7073-10]

L. Bivolarski, Logitech, Inc. (United States)

\section{SESSION $3 \quad$ ADVANCED VIDEO CODING}

7073 OC Low-complexity hierarchical lapped transform for lossy-to-lossless image coding in JPEG XR / HD Photo [7073-12]

C. Tu, S. Srinivasan, G. J. Sullivan, S. Regunathan, H. S. Malvar, Microsoft Corp. (United States) 
7073 OD SVC overview and performance evaluation [7073-13]

T. Oelbaum, Technische Univ. München (Germany); H. Schwarz, Fraunhofer Institute for Telecommunications-HHI (Germany); M. Wien, RWTH Aachen Univ. (Germany); T. Wiegand, Fraunhofer Institute for Telecommunications-HHI (Germany) and Technische Univ. Berlin (Germany)

$7073 \mathrm{OE} \quad$ Low complexity inter-layer residual coding and efficient mode decision for spatial and temporal scalable video coding [7073-14]

B. Lee, T. Na, M. Kim, Information and Communications Univ. (Korea, Republic of); S. Hahm, C. Park, I. Cho, Technical Research Institute, Korea Broadcasting System (Korea, Republic of)

7073 OF Toward a 3D video format for auto-stereoscopic displays [7073-15] A. Vetro, S. Yea, Mitsubishi Electric Research Labs. (United States); A. Smolic, Fraunhofer Institute for Telecommunications-Heinrich-Hertz-Institut (Germany)

7073 OG High-throughput architecture for H.264/AVC CABAC encoding and decoding system [7073-16]

Y.-T. Chang, Industrial Technology Research Institute (Taiwan)

$7073 \mathrm{OH} \quad$ Modern transform design for advanced image/video coding applications [7073-17]

T. D. Tran, FastVDO, Inc. (United States) and Johns Hopkins Univ. (United States);

P. N. Topiwala, FastVDO, Inc. (United States)

7073 Ol A fast macroblock mode decision scheme using ROI-based coding of H.264/MPEG-4 Part 10 AVC for mobile video telephony applications [7073-18]

T. Na, Y. Lee, Information and Communications Univ. (Korea, Republic of); J. Lim, Y. Joo, K. Kim, J. Byun, SK Telecom (Korea, Republic of); M. Kim, Information and Communications Univ. (Korea, Republic of)

7073 OJ Apply network coding for H.264/SVC multicasting [7073-19]

H. Wang, C.-C. J. Kuo, Univ. of Southern California, Los Angeles (United States)

\section{SESSION 4 MOBILE VIDEO AND APPLICATIONS}

$7073 \mathrm{OL} \quad$ Error resiliency of distributed video coding in wireless video communication [7073-21] S. Ye, M. Ouaret, F. Dufaux, M. Ansorge, T. Ebrahimi, École Polytechnique Fédérale de Lausanne (Switzerland)

$70730 \mathrm{M}$ Temporal flicker reduction and denoising in video using sparse directional transforms [7073-22]

S. Kanumuri, O. G. Guleryuz, M. R. Civanlar, DoCoMo Communications Labs. USA, Inc. (United States); A. Fujibayashi, C. S. Boon, NTT DoCoMo, Inc. (Japan)

7073 ON Exploiting spatio-temporal characteristics of human vision for mobile video applications [7073-23]

R. Jillani, H. Kalva, Florida Atlantic Univ. (United States)

$707300 \quad$ Mobile video processing for visual saliency map determination [7073-24]

S. XU, Huazhong Univ. of Science and Technology (China); W. Lin, Nanyang Technological Univ. (Singapore); C.-C. J. Kuo, Univ. of Southern California, Los Angeles (United States) 
7073 OP Scorebox extraction from mobile sports videos using Support Vector Machines [7073-25] W. Kim, J. Park, C. Kim, Information and Communications Univ. (Korea, Republic of)

$70730 Q$ Sub-component modeling for face image reconstruction in video communications [7073-26]

D. J. Shiell, J. Xiao, Epson Research \& Development, Inc. (United States); A. K. Katsaggelos, Northwestern Univ. (United States)

7073 OS Video quality measure for mobile IPTV service [7073-28]

W. Kim, C. Kim, Information and Communications Univ. (Korea, Republic of)

\section{SESSION 5 RATE CONTROL FOR VIDEO SYSTEMS}

7073 OT Rate-controlled requantization transcoding for H.264/AVC video streams [7073-29]

S. Notebaert, J. De Cock, P. Lambert, R. Van de Walle, Ghent Univ. (Belgium)

7073 OU Efficient network-aware macroblock mode decision for error resilient H.264/AVC video coding [7073-30]

L. D. Soares, P. Nunes, Instituto Superior de Cilências do Trabalho e da Empresa-Instituto de Telecomunicações (Portugal); F. Pereira, Instituto Superior Técnico-Instituto de Telecomunicações (Portugal)

7073 OW Rate controlling for color and depth based 3D video coding [7073-32]

B. Kamolrat, W. A. C. Fernando, M. Mrak, Univ. of Surrey (United Kingdom)

\section{SESSION 6 PERFORMANCE EVALUATION IN IMAGE CODING APPLICATIONS}

7073 OX Toward objective image quality metrics: the AIC eval program of the JPEG [7073-34] T. Richter, Univ. Stuttgart (Germany); C. Larabi, Univ. of Poitiers, XLIM-SIC, UMR CNRS 6172 (France)

$70730 Z$ A comparative study of color image compression standards using perceptually driven quality metrics [7073-36]

F. De Simone, D. Ticca, F. Dufaux, M. Ansorge, T. Ebrahimi, École Polytechnique Fédérale de Lausanne (Switzerland)

707311 Application specific performance measurements of image compression codecs [7073-38] T. Richter, Univ. Stuttgart (Germany)

SESSION 7 INITIATIVES IN IMAGE CODING AND ACCESSORIES AND APPLICATIONS

707312 Image coding design considerations for cascaded encoding-decoding cycles and image editing: analysis of JPEG 1, JPEG 2000, and JPEG XR / HD Photo [7073-39]

G. J. Sullivan, S. Sun, S. Regunathan, D. Schonberg, C. Tu, S. Srinivasan, Microsoft Corp. (United States)

707313 ITU-T T.851: an enhanced entropy coding design for JPEG baseline images [7073-40] J. L. Mitchell, A. T. Hinds, Ricoh/IBM InfoPrint Solutions Co. (United States) 
707314 Techniques for enhancing JPEG XR / HD Photo rate-distortion performance for particular fidelity metrics [7073-41]

D. Schonberg, S. Sun, G. J. Sullivan, S. Regunathan, Z. Zhou, S. Srinivasan, Microsoft Corp. (United States)

707315 Coding of high dynamic range images in JPEG XR / HD Photo [7073-42]

S. Srinivasan, Z. Zhou, G. J. Sullivan, R. Rossi, S. Regunathan, C. Tu, A. Roy, Microsoft Corp. (United States)

707316 Split field coding: low complexity error-resilient entropy coding for image compression [7073-43]

J. J. Meany, C. J. Martens, Boeing (United States)

707317 Scalable low complexity image coder for remote volume visualization [7073-44]

H. G. Lalgudi, M. W. Marcellin, A. Bilgin, The Univ. of Arizona (United States); M. S. Nadar, Siemens Corporate Research (United States)

\section{SESSION $8 \quad$ BIOMEDICAL APPLICATIONS}

707319 Hyperspectral imaging with wavelet transform for classification of colon tissue biopsy samples [7073-49]

K. Masood, Univ. of Warwick (United Kingdom)

\section{OPTICAL AND DIGITAL IMAGE PROCESSING SYSTEMS}

7073 1A Optical flow processing for sub-pixel registration of speckle image sequences [7073-50] C. Cofaru, W. Philips, Ghent Univ. Telin-IPI-IBBT (Belgium); W. Van Paepegem, Ghent Univ. (Belgium)

7073 1B Advanced super-resolution image enhancement process [7073-51]

H.-W. Chen, D. Braunreiter, Science Applications International Corp. (United States)

7073 1C Fibered fluorescence microscopy (FFM) of intra epidermal nerve fibers--translational marker for peripheral neuropathies in preclinical research: processing and analysis of the data [7073-52]

F. Cornelissen, Janssen Pharmaceutica (Belgium); S. De Backer, Antwerp Univ. (Belgium);

J. Lemeire, Vrije Univ. Brussel (Belgium); B. Torfs, R. Nuydens, T. Meert, Janssen

Pharmaceutica (Belgium); P. Schelkens, Vrije Univ. Brussel (Belgium); P. Scheunders,

Antwerp Univ. (Belgium)

7073 1D Algorithm design of liquid lens inspection system [7073-54]

L.-L. Hsieh, C.-C. Wang, Industrial Technology Research Institute (Taiwan)

$70731 \mathrm{G} \quad$ Parallel magnetic resonance imaging using compressed sensing [7073-57] A. Bilgin, Y. Kim, H. G. Lalgudi, T. P. Trouard, M. I. Altbach, The Univ. of Arizona (United States) 
707311 SAR automatic target recognition using maximum likelihood template-based classifiers [7073-59]

J. A. Saghri, California Polytechnic State Univ. (United States)

7073 i Automatic generation of $\mathbf{3 6 0}$ degree panorama from image sequences [7073-60]

S. Ho, P. David, Army Research Lab. (United States)

\section{SESSION 10 IMPLEMENTATION AND PROCESSING SCENARIOS III}

$70731 \mathrm{~K}$ Implementation and evaluation of real-time pan-tilt-zoom camera calibration [7073-61] N. Fung, P. David, Army Research Lab. (United States)

7073 1L Adaptive fingerprint enhancement and identification using linear parametric models [7073-62]

M. Mehrübeoğlu, Texas A\&M Univ., Corpus Christi (United States); L. McLauchlan, Texas A\&M Univ., Kingsville (United States)

$70731 \mathrm{M}$ Design and evaluation of sparse quantization index modulation watermarking schemes [7073-63]

B. Cornelis, J. Barbarien, A. Dooms, A. Munteanu, J. Cornelis, P. Schelkens, Vrije Univ. Brussel (Belgium)

7073 iN Acquisition and registration of aerial video imagery of urban traffic [7073-64]

R. C. Loveland, E. Rosten, Los Alamos National Lab. (United States)

707310 High speed acquisition system of photo-colorimetric images to record and to model human visual signal [7073-67]

V. Boucher, F. Greffier, F. Fournela, Lab. Régional des Ponts et Chaussées d'Angers (France)

POSTER SESSION

7073 IP Restoration of atmospherically degraded images using a sparse prior [7073-68]

Z. Wen, D. Fraser, A. Lambert, ITEE, ADFA/UNSW (Australia)

7073 IR Efficient video segmentation using temporally updated mean shift clustering [7073-72] N. Petrović, L. Jovanov, A. Pižurica, W. Philips, Ghent Univ. (Belgium)

7073 is Wavelet and PCA-based approach for 3D shape recovery from image focus [7073-73] M. T. Mahmood, S. Shim, T.-S. Choi, Gwangju Institute of Science and Technology (Korea, Republic of)

7073 IT Optimization of focus measure using genetic algorithm [7073-74]

I.-H. Lee, M. T. Mahmood, T.-S. Choi, Gwangju Institute of Science and Technology (Korea, Republic of)

7073 IU Fast three-dimensional shape recovery in TFT-LCD manufacturing [7073-75]

W.-J. Choi, T.-S. Choi, Gwangju Institute of Science and Technology (Korea, Republic of) 
7073 IV Using wavelets for edge directed image interpolation [7073-76]

E. P. Lam, Thales Raytheon Systems (United States)

7073 IW A novel multiscale segmentation method for HRCT images [7073-77]

S.-C. Park, T.-S. Choi, Gwangju Institute of Science and Technology (Korea, Republic of)

$707312 \quad$ Estimating surface roughness using image focus [7073-80]

A. S. Malik, T.-S. Choi, Gwangju Institute of Science and Technology (Korea, Republic of)

707320 Estimation of depth map based on focus adjustment [7073-81]

S.-O. Shim, A. S. Malik, M. T. Mahmood, T.-S. Choi, Gwangju Institute of Science and Technology (Korea, Republic of)

707321 A fast multi-pattern motion estimation algorithm based on the nature of error surfaces [7073-82]

H. Nisar, T.-S. Choi, Gwangju Institute of Science and Technology (Korea, Republic of)

707322 Image restoration based on camera microscanning [7073-83]

J. L. López-Martínez, V. Kober, Ctr. de Investigación Científica y de Educación Superior de Ensenada (Mexico)

707323 Remote counseling using HyperMirror quasi space-sharing system [7073-84]

S. Hashimoto, Univ. of Tsukuba (Japan) and National Institute of Information and Communications Technology (Japan); O. Morikawa, National Institute of Advanced Industrial Science and Technology (AIST) (Japan) and National Institute of Information and Communications Technology (Japan); N. Hashimoto, Citizen holdings Co., Ltd. (Japan);

T. Maesako, Osaka Univ. (Japan) and National Institute of Information and Communications Technology (Japan)

707324 Alignment of vectorial shearing interferometer using a simple recognition algorithm [7073-85]

G. Garcia-Torales, J. L. Flores, Univ. de Guadalajara (Mexico); J. Álvarez-Borrego, Ctr. de Investigación Científica y de Educación Superior de Ensenada (Mexico)

707325 System of invariant correlation to rotation using a ring mask [7073-86]

S. Solorza, Univ. Autónoma de Baja California (Mexico); J. Álvarez-Borrego, V. Kober, Ctr. de Investigación Científica y de Educación Superior de Ensenada (Mexico)

707326 Invariant correlation of objects using non-linear composite filters [7073-87]

R. E. Guerrero-Moreno, J. Álvarez-Borrego, Ctr. de Investigación Científica y de Educación Superior de Ensenada (Mexico)

707327 Distortion-invariant pattern recognition with nonlinear correlation filters [7073-88]

S. Martínez-Díaz, V. Kober, CISESE (Mexico)

707328 Optical inspection for electronic assemblies using nonlinear correlation filters [7073-89] J. L. Flores, G. García-Torales, Univ. de Guadalajara (Mexico); J. Álvarez Borrego, Ctr. de Investigación Científica y de Educación Superior de Ensenada (Mexico) 
707329 Invariant recognition to position, rotation, and scale considering vectorial signatures [7073-90]

J. R. Lerma A., Univ. Autónoma de Baja California (Mexico); J. Álvarez-Borrego, Ctr. de Investigación Científica y de Educación Superior de Ensenada (Mexico);

J. Á. González-Fraga, Univ. Autónoma de Baja California (Mexico)

7073 2A Motivation and implementation of a software SVC real-time VGA encoder for mobile TV [7073-91]

V. Bottreau, T. Viellard, C. Chevance, O. Bureller, Thomson R\&D France (France)

7073 2B Digital holographic video of plankton [7073-92]

V. V. Dyomin, A. S. Olshukov, Tomsk State Univ. (Russia)

$70732 \mathrm{C} \quad$ Study on depth of field of wavefront coding imaging system [7073-93]

C. Pan, Univ. of Shanghai for Science and Technology (China) and Huangshi Institute of Technology (China); J. Chen, D. Zhang, S. Zhuang, Univ. of Shanghai for Science and Technology (China)

$70732 \mathrm{E} \quad$ An efficient algorithm based on dynamic programming for 3D shape recovery [7073-95] L. M. Jimenez-Medina, V. Kober, H. H. Hidalgo-Silva, Ctr. de Investigación Científica y de Educación Superior de Ensenada (Mexico)

$70732 \mathrm{~F} \quad$ Application of digital image processing method for fish age estimation [7073-96] T. WU, J. Hong, South China Univ. of Technology (China)

$70732 \mathrm{H} \quad$ Nonlinear filter for pattern recognition using the scale transform [7073-98]

A. Coronel-Beltrán, Univ. de Sonora (Mexico); J. Álvarez-Borrego, Ctr. de Investigación Científica y de Educación Superior de Ensenada (Mexico)

707321 Ordinal-based method for robust image/video signature generation [7073-100]

D. C. Chen, L. Chaisorn, S. Rahardja, A*STAR Institute for Infocomm Research (Singapore)

7073 2J Large-scale 3-D profilometer [7073-101]

Y.-Y. Lan, J.-L. Chen, W.-C. Wang, L.-R. Chang, Industrial Technology Research Institute (Taiwan)

Author Index 
Downloaded From: https://www.spiedigitallibrary.org/conference-proceedings-of-spie on 26 Apr 2023

Terms of Use: https://www.spiedigitallibrary.org/terms-of-use 


\title{
Conference Committee
}

\author{
Program Track Chair
}

Khan M. Iftekharuddin, University of Memphis (United States)

Conference Chair

Andrew G. Tescher, AGT Associates (United States)

Program Committee

Vasudev Bhaskaran, Marvell Semiconductor, Inc. (United States)

Bernard V. Brower, Eastman Kodak Company (United States)

Wo L. Chang, National Institute of Standards and Technology (United States)

Touradj Ebrahimi, École Polyłechnique Fédérale de Lausanne (Switzerland) and Emitall S.A. (Switzerland)

Ali Habibi, The Aerospace Corporation (United States)

Arianne T. Hinds, InfoPrint Solutions Company (United States)

T. Russell Hsing, Telcordia Technologies, Inc. (United States)

Kristina M. Johnson, Johns Hopkins University (United States)

C.-C. Jay Kuo, University of Southern California (United States)

Catherine Lambert-Nebout, Centre National d'Études Spatiales (France)

Andre J. Oosterlinck, Katholieke Universiteit Leuven (Belgium)

Sethuraman Panchanathan, Arizona State University (United States)

Fernando Pereira, Instituto Superior Técnico (Portugal)

Robert A. Rossi, Jr., Microsoft Corporation (United States)

John A. Saghri, California Polytechnic State University (United States)

Peter Schelkens, Vrije Universiteit Brussel (Belgium)

Gary J. Sullivan, Microsoft Corporation (United States)

Pankaj Topiwala, FastVDO LLC (United States)

Mihaela van der Schaar, University of California, Los Angeles (United States)

\section{Session Chairs}

1 Implementation and Processing Scenarios I

Touradj Ebrahimi, Ecole Polytechnique Fédérale de Lausanne

(Switzerland)

2 Design and Implementation of Color Transformations

Arianne T. Hinds, InfoPrint Solutions Company (United States) 
3 Advanced Video Coding

Pankaj Topiwala, FastVDO LLC (United States)

$4 \quad$ Mobile Video and Applications

Vasudev Bhaskaran, Marvell Semiconductor, Inc. (United States)

5 Rate Control for Video Systems

Fernando Pereira, Instituto Superior Técnico (Portugal)

6 Performance Evaluation in Image Coding Applications

Gary J. Sullivan, Microsoft Corporation (United States)

$7 \quad$ Initiatives in Image Coding and Accessories and Applications

Touradj Ebrahimi, École Polytechnique Fédérale de Lausanne (Switzerland)

8 Biomedical Applications

Peter Schelkens, Vrije Universiteit Brussel (Belgium)

9 Optical and Digital Image Processing Systems

Peter Schelkens, Vrije Universiteit Brussel (Belgium)

10 Implementation and Processing Scenarios II

John A. Saghri, California Polytechnic State University (United States)

11 Implementation and Processing Scenarios III

John A. Saghri, California Polytechnic State University (United States) 